\title{
Green fluorescent protein labeling of dopaminergic neurons in zebrafish for the study of parkinson's
} disease

\begin{abstract}
The loss of dopaminergic neurons in the substantia nigra is the pathological hallmark of Parkinson's disease (PD) associated with the characteristic motor deficits. Despite all the recent progress in the identification of genes and pathways associated with PD, we still lack a complete understanding of the molecular mechanisms underpinning the disease. Our goals were to develop a new transgenic zebra fish line where tyrosine hydroxylase (TH)positive neurons were labeled with green fluorescent protein (EGFP) under the control of the zebra fish tyrosine hydroxylase (th1) promoter and to demonstrate the usefulness of the model for the study of the mechanisms underlying cell death in a toxin induced model of $\mathrm{PD}$
\end{abstract}

A $2.5 \mathrm{~kb}$ gene fragment of the th 1 promoter was isolated and cloned into a mini-Tol2vecto followed by EGFP and microinjected to originate the Tg (-2.5th: EGFP) zebra fish line. Tg (-2.5th: EGFP) were visualized by fluorescence microscopy for EGFP and TH expression during development. The levels of EGFP mRNA were detected by RT-qPCR and protein expression was examined by western blot analysis. The model of neurotoxicity was generated by treating transgenic zebra fish with 6-hydroxydopamine (6-OHDA), followed by the analysis of cell death markers and behavior.

EGFP expression was detected mainly in the posterior tuberculum of the ventral diencephalon (vDC). In the vDC, TH-labeled neurons of groups 1-were correctly labeled with EGFP. Further, we demonstrated that these neurons in larvae and adults are sensitive to 6-OHDA treatment, resulting in cell loss and behavioral deficits. In conclusion, this transgenic line constitutes a powerful tool to visualize and study dopaminergic neurons throughout the zebra fish life-cycle and enables high-throughput screening of molecules of interest for PD.

Keywords: parkinson's disease, dopaminergic neurons, transgenic zebra fish
Volume 4 Issue I - 2017

\author{
Ana Dulce Correia,' Rui S Soares,' Karina \\ Rahimi, ${ }^{2}$ Sara Sousa, ${ }^{3}$ Tiago F Outeiro, ${ }^{1,4}$ \\ Nuno Afonso, ${ }^{3}$ Rob Willemsen, ${ }^{2}$ Herma van \\ der Linde ${ }^{2}$ \\ 'Department of Cell and Molecular Neuroscience Unit, Instituto \\ de Medicina Molecular, Portugal, Europe \\ ${ }^{2}$ Department of Clinical Genetics, Erasmus MC, Rotterdam, \\ Netherlands \\ ${ }^{3}$ Techno Phage SA, Portugal \\ ${ }^{4}$ Department of Neuro degeneration and restorative research, \\ University Medical Center Goettingen, Germany
}

\begin{abstract}
Correspondence: Ana Dulce Correia, PhD., Battelle UK, 29 Springfield Lyons Approach, Springfield, Chelmsford, CM2 5LB, Tel 00440 I 245 450777, Fax 00440 I 245 397900,

Email correia.ana10@gmail.com
\end{abstract}

Received: October 27, 2016 | Published: February 01, 2017

\section{Introduction}

Parkinson's disease (PD) is a progressive neurodegenerative disorder that is characterized by motor and non-motor manifestations that are progressive and ultimately refractory to therapeutic interventions. ${ }^{1,2}$ Dopamine is an important catecholamine neurotransmitter that has been linked with several neurological diseases in humans. ${ }^{3}$ The loss of dopaminergic neurons emanating from the substantia nigra in humans is largely responsible for the motor manifestations seen in PD. ${ }^{4}$ Despite of anatomical and functional studies of the dopaminergic system in different species have contributed to valuable insights into the pathogenesis of $\mathrm{PD},{ }^{5}$ the understanding of the molecular mechanisms underlying this disease has been hampered by the limitations of the existing animal models. ${ }^{6}$

The teleost, zebra fish (Danio rerio) has proven to be an excellent model organism for developmental genetic studies and recently has emerged as a valuable model to study of different aspects associated with neuro degeneration. ${ }^{7,8}$ As a vertebrate, the basic organization and divisions of the CNS are similar to those of other vertebrates and specialized neuronal populations, including the dopaminergic system also appear well conserved. ${ }^{9}$ The DA clusters in CNS related to substantia nigra region in humans are not seen in the midbrain of zebra fish but several neuronal groups exist in the posterior tuberculum (PT) of the ventral diencephalon (vDC) that has been shown to be the human equivalent. ${ }^{10}$ In particular, the TH populations 2 to 6 have ascending projections to the subpallium of telencenphalon, considered to be similar to the dopaminergic projections to the striatum of mammals. ${ }^{10,11}$ Treatment with Parkinsonism-inducing neurotoxins, such as MPTP or 6-OHDA induces the loss of DA neurons in vDC and pretectum $(\mathrm{Pr})$ along with aberrant swimming patterns. ${ }^{11-14}$

Several attempts have been made to generate transgenic lines expressing reporter genes in DA neurons in the zebra fish brain. Specifically, the first lines were generated with constructs containing $\mathrm{TH}$ promoter that could only drive GFP expression in retinal cells. ${ }^{15,16}$ Despite the diverse role of DA cells in retinal functions, the understanding of DA cell function has been limited because they have a low density in the retina and cannot be identified in living retina by morphological characteristics. However, the use of other promoters (e.g., dat and vmat2) have contributed successfully for the understanding of the role of different monoaminergic neurons in the brain, ${ }^{11}$ including the dopaminergic neurons (DA) located in the diencenphalon. ${ }^{8,17}$ In spite of this, the DA cell function is not completely understood and it could benefit from the development of other transgenic zebra fish models with increased labeling of DA neurons in the brain.

In this study, we developed a novel transgenic zebra fish line expressing enhanced green fluorescent protein (EGFP) in THpositive neurons. Next, we used this Tg line in order to investigate the effects of 6-OHDA on neuronal loss and in inducing swimming abnormalities. Thus, this zebra fish line constitutes a powerful tool to study DA neurons in vivo and to perform screens for agents protecting against DA-cell loss which might be used for the development of novel therapeutic approaches for PD and other disorders associated with the loss of DA neurons. 


\section{Materials and methods}

\section{Zebra fish handling and growth conditions}

Wild-type zebra fish (TU strain) were obtained from ZIRC (University of Oregon, Eugene, USA). All zebra fish were maintained and bred in similar culture conditions by following standard guidelines for fish care and maintenance protocols. ${ }^{18}$ Developmental stages were determined according to. ${ }^{19}$ This study was carried out in strict accordance with the recommendations in the Guide for the Care and Use of Laboratory Animals of the National Institutes of Health. The protocol was approved by the Animal Ethics Committee of the Instituto de Medicina Molecular (Permit Number: AEC 0042009 ACorreia). All surgery was performed under tricaine anesthesia, and all efforts were made to minimize suffering and reduce the number of animals used.

\section{Generation of the transgenic zebra fish line}

A $2.5 \mathrm{~kb}$ upstream promoter region of th1 was amplified by PCR from genomic DNA from Zebra fish (AB strain) using the following primers: F: GCAGATTGAATGAAAGAAGTGG (ATG-2.501) and R: TGCTTGAATTCGGCTTGTTCAG. The ATG (reverse complement: CAT) was replaced by AAG (reverse complement: CTT). The EcoRI site (GAATTC) was used for cloning (Figure 1A). The PCR product was cloned into a mini-Tol2 vector followed by EGFP and a SV40 PolyA signaling. ${ }^{20}$

The linearized plasmid $(30 \mu \mathrm{g} / \mu \mathrm{l})$ and tol 2 transposase mRNA $(25 \mu \mathrm{g} / \mu \mathrm{l})$ were co-injected into one-cell stage of fertilized wild-type zebra fish eggs. The DNA-injected embryos were examined for in vivoEGFP expression at 48 hours post fertilization (hpf). Those that expressed EGFP at a high level in the neuronal cells were raised to adulthood (F0 founders). Founders were selected and out crossed with wild-type fish and the F1 progeny screened for germline transmission. Adult F1 fish were then out crossed to establish a stable transgenic line (F2). To verify whether the transgene was integrated, F2 fish were genotyped following the protocol described by. ${ }^{21}$

Embryos were regularly monitored for in vivo EGFP expression using a fluorescence stereomicroscope (Leica MZ10F) connected to a Media Cybernetics Evolution MP Color digital camera. The brightfield or fluorescent images were captured using Quantitative Imaging Corporation Q-Capture software. Live images here were taken from treated transgenic embryos with 1-phenyl-2-thiourea $(0.003 \%$ phenylthiourea, Sigma, Dorset, UK) to prevent pigment formation.

\section{RT-qPCR analysis of EGFP mRNA}

Total RNA was extracted from snap-frozen pooled larvae or brain tissue (30 mg) using a column isolation kit (Rneasy mini Kit, Qiagen, Valencia, CA) and treated with DNase (RQ1 DNase, Promega, Madison, WI), according to the manufacturer's instructions. RNA integrity and total RNA concentration were assessed using a NanoDrop ND-1000 spectrophotometer (NanoDrop technologies, Wilmington, DE). $300 \mathrm{ng}$ of total RNA was reverse transcribed using the iScript ${ }^{\mathrm{TM}}$ Reverse Transcription Super mix for RT-qPCR (Biorad, Hercules, CA, USA). Relative EGFP expression was quantified in 1 $\mu 1$ of cDNA by real-time qPCR in $20 \mu 1$ reactions containing $10 \mu 1$ of DyNAmo SYBR Green Super Mix (Biorad, Hercules, CA, USA) and 4 pmol of each primer (EGFPq2F: GAGCTGAAGGGCATCGACTT and EGFPq2R: CCATGATATAGACGTTGTGGCTG ). Ef1a was used as the endogenous control to normalize gene expression data (eflF: CTGGAGGCCAGCTCAAACAT and eflR: ATCAAGAAGAGTAGTACCGCTAGCATTAC). Samples were assayed in duplicate in runs consisting of a initial stage at $95{ }^{\circ} \mathrm{C}$ for
10 minutes, followed by 40 cycles at $95^{\circ} \mathrm{C}$ for 15 seconds and $60{ }^{\circ} \mathrm{C}$ for 1 minute. All RT-qPCR was conducted using an ABI Prism 7500 instrument (Applied Biosystems, Germany).

\section{Western blot analysis}

Zebra fish larvae and brain tissue were suspended in Fish Ringer's buffer $116 \mathrm{mM} \mathrm{NaCl}, 2.9 \mathrm{mM} \mathrm{KCl}, 1.8 \mathrm{mM} \mathrm{CaCl} 2,5 \mathrm{mM}$ HEPES, pH 7.2 and 1 mM EDTA plus protease inhibitor cocktail (Sigma, UK) or CHAPS buffer $10 \mathrm{mM}$ TRIS-HCl pH 7.5, $1 \mathrm{mM} \mathrm{MgCl2,} 1 \mathrm{mM}$ EGTA, $0.1 \mathrm{mM}$ Benzamidine, 0.5\% CHAPS and 10\% Glycerol plus protease inhibitor cocktail (Sigma, UK) respectively and lysed by sonication for 20 seconds. Protein homogenates were then centrifuged $\left(20,000 \times \mathrm{g}\right.$ for 30 minutes at $\left.4^{\circ} \mathrm{C}\right)$ and protein concentration was measured in the supernatants by standard Bradford Assay (Biorad, Hercules, CA, USA). Samples (20 $\mu \mathrm{g}$ for larvae or $15 \mu \mathrm{g}$ for brain tissue) were added to $4 \mu$ lof sample buffer, heated at $95^{\circ} \mathrm{C}$ for 5 minutes and separated on $10 \%$ polyacrilamyde gel. Proteins were transferred to Immun-Blot PVDF membrane (Biorad, USA) and blocked for 1 hour in 5\% milk TRIS-buffered saline- $0.05 \%$ Tween-20 (TBS-T). For analyses, membranes were incubated overnight at $4{ }^{\circ} \mathrm{C}$ with selected primary antibodies differently diluted (in 3\% BSA and $0.02 \%$ $\mathrm{NaNO}_{3}$ in TBST) for larvae and brain homogenates respectively): anti-TH (ab112, Abcam, USA) used at 1:4,000 or 1:20,000; anti$\beta$-actin (AM4302, Applied Biosystems, USA) used at 1:5,000 or 1:100,000 and anti-GFP (sc-9996, Santa Cruz Biotechnology, USA) used at 1:10,000. Samples were washed and incubated for 1 hour with peroxidase conjugated secondary antibodies goat anti-mouse IgGHRP (sc-2005, Santa Cruz Biotechnology, USA) and goat anti-rabbit IgG-HRP (sc-2004, Santa Cruz Biotechnology, USA) diluted 1:10,000 in 5\% milk in TBS-T. Immunoreactive bands were detected using enhanced chemiluminescence (ECL Kit, Amersham, UK). Bands were quantified by densitometry (Image J v1.45s, National Institutes of Health, USA). TH and GFP quantification was normalized for protein loading using the $\beta$-actin protein expression values.

\section{Whole-mount in situ hybridization and quantification of TH positive cells}

A partial sequence of the th1 cDNA was amplified by PCR, using the following primers $\mathrm{F}(\mathrm{ex} 1)$ :CCACAAAGAGCATCCGGAG and $\mathrm{R}$ (ex7): CTCGGAGGGTGGAGTAGA. The $669 \mathrm{bp}$ PCR product was cloned in the pCRII-TOPO vector (K4650-40, Invitrogen). Whole mount in situ hybridization was performed with digoxigenin (DIG)labeled RNA probes as previously described. ${ }^{22}$ Briefly, a digoxigenin labeled antisense RNA probe was synthesized from the plasmid containing a part of the thcDNA linearized by BamHI (10656275001, Roche, Switzerland) and transcribed using T7 RNA polymerase (18033-019, Invitrogen, USA) and the DIG RNA Labeling Mix (11277073910, Roche, Switzerland). Wild-type and transgenic embryos were fixed at 3-dpf overnight in 4\% paraformaldehyde (PFA) in phosphate-buffered saline (PBS), then transferred to $100 \%$ methanol and stored at $-20^{\circ} \mathrm{C}$ in $100 \%$ methanol until use. The hybridization procedure was performed as described, ${ }^{22}$ embryos were washed extensively in $0.1 \%$ Tween 20 in PBS, re-fixed in 4\% PFA in PBS, then transferred to $80 \%$ glycerol. Embryos were mounted in glycerol and TH-positive diencephalic neurons counted under an Olympus SZX16 microscope. In order to avoid introducing unintended bias, all embryos were coded and scored blind. The results are expressed as average number of neurons present in wild-type embryos, which were staged and treated in parallel with transgenic embryos.

\section{Immunohistochemistry}

Larvae for double immunohistochemistry were fixed in $4 \% \mathrm{PFA} / 1$ $x$ PBS for 1, 5 hours at room temperature (RT), rinsed in 1xPBS and 
placed $30 \%$ sucrose $/ 1 \times$ PBS to equilibrate. Larvae were then embedded in gelatin, sectioned on Leica (Cryostat LEICA CM 3050S) cryostat at $14 \mu \mathrm{m}$ and stored at $-20^{\circ} \mathrm{C}$ until use. After $3 \mathrm{x}$ washes in $1 \mathrm{xPBS}$ for 10 minutes, the sections were pre-incubated with blocking solution containing 5\% goat serum in PBST for 1,5 hours at RT and then incubated with the same blocking solution containing the primary antibodies (see below). After removing the antibodies with 3 washes in $1 x$ PBS, the appropriate secondary antibodies were incubated on the slides for 2 hours at RT. After thoroughly washing, fluorescence images were recorded by confocal laser scanning microscopy (Zeiss LSM 510 META). The following primary and secondary antibodies were used: mouse anti-GFP (1:500, Sc9996, Santa Cruz, USA), rabbit anti-TH (1:300, ab112, Abcam, USA), rabbit anti-caspase-3 (1:1000, ab13847, Abcam, USA), goat anti-mouse Alexa Fluor 488 (1:500, A11001, Invitrogen, USA) and goat anti-rabbit Alexa Fluor 568 (1:500, A11011, Invitrogen, USA).

Adult brains for double immunohistochemistry were fixed by $4 \% \mathrm{PFA} / 1 \mathrm{x} \mathrm{PBS}$ overnight at $4^{\circ} \mathrm{C}$, following standard procedures. The analyses of sections were performed by standard methods. Briefly, embryos and adults brains were fixed, dehydrated, embedded in paraffin, and sectioned $(7 \mu \mathrm{m})$. Sections were deparaffinized, blocked, antigen-retrieved, incubated overnight with the selected primary antibodies: mouse anti-GFP used at 1:500 (1814460, Roche, Switzerland); rabbit anti-TH used at 1:100 (AB152, Chemicon, USA) dilutions. Sections were then washed and incubated with the secondary antibodies: anti-mouse Cy3 used at 1:100 (715-165-150, Jackson, USA) and anti-rabbit Alexa Fluor $488 \mathrm{~F}(\mathrm{ab}) 2$ used at 1:100 (A11070, Invitrogen, USA). The cross-species reactivity of the used antibodies was confirmed. Fluorescence images were recorded with an epifluorescence microscope (Zeiss Axiovert 200M).

\section{6-OHDA treatments}

A master stock solution of 6-hydroxydopamine hydrochloride (6OHDA, Sigma-Aldrich, USA) was prepared in $0.2 \%$ ascorbic acid solution (analytical grade, Sigma) and stored at $-20^{\circ} \mathrm{C}$. This stock solution was used to prepare all working solutions in experiments with larvae and adult transgenic zebra fish.

Effects of 6-OHDA on the swimming activity of larvae zebra fish were determined by 3-day waterborne tests. Larvae zebra fish, $\mathrm{Tg}(-$ 2.5th:EGFP) were exposed to a range of concentrations of 6-OHDA $(250 \mu \mathrm{M}$ to $2000 \mu \mathrm{M})$ for 2 days to study preliminary effects of this drug on embryo survival. The concentration $500 \mu \mathrm{M}$ of 6-OHDA was chosen because significantly has both reduced brain EGFP expression and promoted differences in locomotors larvae behavior. Briefly, transgenic embryos were obtained from mating pairs of homozygous $\mathrm{Tg}\left(-2.5\right.$ th:EGFP) fish and raised at $28.5^{\circ} \mathrm{C}$ in $\mathrm{E} 3$ medium $(5 \mathrm{mM} \mathrm{NaCl}$, $0.17 \mathrm{mM} \mathrm{KCl}, 0.33 \mathrm{mM} \mathrm{CaCl}_{2}, 0.33 \mathrm{mM} \mathrm{MgSO}_{4}$ ). At $3 \mathrm{dpf}$, embryos were treated with $500 \mu \mathrm{M}$. Control groups are kept in E3 medium. After 3 days of exposure, groups of treated embryos including controls were randomly examined for in vivo EGFP expression using a fluorescence microscope (Zeiss Axiovert 200M microscope coupled to a sensitive cooled CCD camera, Roper Scientific Coolsnap HQ $\mathrm{CCD}$ ) and analyzed with Image J software. The embryos were then fixed in 4\% PFA for immunohistochemical analyses. Locomotors behavioral analyses were performed on treated $(n=8)$ and control $(\mathrm{n}=8)$ embryos at day $6 \mathrm{dpf}$ using a DanioVision ${ }^{\mathrm{TM}}$ automated tracking system for larvae zebra fish from Noldus Information Technology (Wageningen, the Netherlands). This experiment was repeated 6 times. Briefly, 6 dpf embryos were analyzed for swimming activity inside the Danio Vision Observation Chamber. The acquired track files were analyzed for total distance moved on periods of 10 minutes (data collected over 30 minutes under stimulus of light and dark) and this procedure was repeated 3 times for each larvae, using Ethovision X.T. 8.5.614 software (Noldus Information Technology, Wageningen, Netherlands). Minimal distance moved filter, was used to filter out system noise (2010 Noldus Information Technology communication).

Effects of 6-OHDA on the motor activity of adult $\operatorname{Tg}(-2.5$ th: EGFP) zebra fish were examined by 9-day behavioral tests. Adult fish $(\mathrm{n}=15)$ were treated intramuscularly (i.m.) with $33 \mathrm{mg} / \mathrm{kg}$ of $6-\mathrm{OHDA}$ and the individual fish swimming responses recorded at various time-points after injections using an electrical biosensor, the MOBS system. ${ }^{23,24}$ The dose, $33 \mathrm{mg} / \mathrm{kg}$, was selected on the bases of literature data and from our pilot experiments. We also tested a control group of fish $(\mathrm{n}=15)$ treated with $0.2 \%$ ascorbic acid (the vehicle solution). The i.m. injections were administrated into anesthetized fish in a total volume of $4.0 \mu \mathrm{l}$ per $0.3 \mathrm{~g}$ fish using a gastight syringe and a 30 -gauge needle (Hamilton, USA). Fish used for the studies had an average body weight of $0.5 \pm 1 \mathrm{~g}$. Before the behavioral tests, small groups of male fish (14-15 animals) were acclimatized to the testing conditions (temperature $22{ }^{\circ} \mathrm{C} \pm 1{ }^{\circ} \mathrm{C}, 10$ hours: 12 hours light-dark cycle) in 17 liter glass aquaria under static conditions and for a minimum of three days. Fish were fed (Sera Vera, Germany) $1 \%$ of body weight per day throughout the tests. On the day of experiments (day 0), either the treated or control groups of fish were individually placed in test chambers for one hour and then individual baseline responses (pre-treatment) were monitored for one hour between $10 \mathrm{a} . \mathrm{m}$ to 15 p.m. Fish were then individually anesthetized with tricaine $(50 \mathrm{mg} / \mathrm{l})$ and were injected with the neurotoxin or the vehicle solution. After injections, fish were kept in extensively aerated water tank until they recovered from the anesthesia. At day 3 fish received a second reinjection with similar volume and dose of neurotoxin. Individual fish swimming responses were recorded used four time-points $(1,3,6$ and 9 days after injections). In each session of analysis the individual responses were evaluated every 15 minutes intervals for a total period of 60 minutes and the outcomes from MOBS reflect the number of tail-flips per minute per individual fish. Analyses with MOBS system contained in total seven-eight independent experiments (total number of fish=30), each including two controls and two fish treated with 6-OHDA and the data was then polled for statistical analyses. After the behavioral recording, fish were humanely sacrificed and brains dissected at days 1, 6 and 9 to measure subcellular responses.

\section{Statistical analyses}

The effect of 6-OHDA on the changes of larvae swimming activity across the recording sessions was analyzed using the unpaired t-student test with Welch's correction. In adults, those comparisons were made using the pair wise, exact Mann-Whitney U-Test. Additional comparisons to day 0 were made using the non-parametric Wilcoxon matched pair test. The level of statistical significance was set to $p<0.05$. Results of other endpoints were analyzed by Student's t-test for independent means. All analyses were performed in StatSoft Statistica ${ }^{\circledR} 6.0$ and GraphPad Prism software packages.

\section{Results}

\section{Generation of the Tg(-2.5th:EGFP) zebra fish line}

In order to generate transgenic zebra fish expressing EGFP in TH-positive neurons, $\sim 500$ embryos were injected with a construct encoding for EGFP under the regulation of a $2.5 \mathrm{~kb}$ fragment of the zebrafish TH promoter. The founders displayed EGFP expression in the brain at $2 \mathrm{dpf}$ and the progeny from outcrosses between the founder fish and wild-type fish showed th-driven EGFP expression was grown to adulthood to establish the transgenic line. Homozygous 
adult fish (F4) were viable and fertile and their morphology was indistinguishable from their wild-type and heterozygote siblings.

Next, we investigated whether the levels of EGFP mRNA and protein were detectable in transgenic larvae and adults. RT-qPCR analyses revealed that homozygous larvae expressed the transcript at 1.3-fold higher levels than the heterozygous siblings. The levels almost quadruplicated in transgenic adult brains. Representative bands of EGFP mRNA levels are shown in Figure 1B. Quantitative western blot analyses were performed in lysates from transgenic larvae and adult animals using a polyclonal anti-GFP antibody. We observed a single band with the expected molecular weight (27 KDa) both in adult brain or larvae zebrafish (Figure 1C).

A diagram showing the DNA fragment used in a mini-Tol2 vector, containing $2.5 \mathrm{~kb}$ of 5'DNA of tyrosine hydroxylase promoter and the cDNA of enhanced green fluorescent protein (EGFP) with SV40 poly-A (pA) tail (\#pEGFP-N1 \#6085-1, Clontech) (A). Polymerase chain reaction (PCR) from reverse transcribed total RNA showing germline transmission of the transgene. Lane1, transgenic (Tg) larvae at 6 days post-fertilization (dpf); lane 2, wild-type (Wt) larvae at $6 \mathrm{dpf}$; lane 3, adult transgenic (Tg) brains; lane 4, adult wild-type (Wt) brains; lanes 5-6, RT controls for genomic DNA contamination in transgenic larvae (-RT larvae) and transgenic brains (-RT Adult), respectively; lane 7, positive controls $(+)$ (EGFP plasmid and efl cDNA). RT-PCR for efl is shown as a loading control (B). Western blot of larvae (Tg and $\mathrm{Wt}$ ) and adult (Tg and $\mathrm{Wt}$ ) zebrafish homogenates probed with anti-tyrosine hydroxylase (TH) and GFP antibodies. Anti-tyrosine hydroxylase antibody was used as the endogenous protein control and $\beta$-actin as the reference control (C).

\section{Transgene expression throughout life-cycle}

To determine the temporal and spatial patterns of expression of the $\operatorname{Tg}(-2.5$ th:EGFP) transgene we assessed the expression of EGFP throughout embryogenesis up to $6 \mathrm{dpf}$. In both F2 and F3 generations we observed EGFP expression in the central nervous system. Specifically, a strong EGFP expression was first detected in the telencephalon (olfactory bulb and preoptic region) at $1 \mathrm{dpf}$ (Figures 2A \& 2B). Progressively, several clusters of EGFP-positive cells were seen scattered in other brain regions mainly in the diencephalon (pretectum, ventral thalamus, posterior tuberculum and hypothalamus) at 3 to $6 \mathrm{dpf}$ (Figures 2C-2F). This pattern of expression persisted in juveniles and was maintained with a strong fluorescent signal in live adult brains (Figures $2 \mathrm{G} \& 2 \mathrm{H}$ ). The EGFP expression was not detected in retina.

At 1-day post-fertilization (dpf), EGFP-positive neurons were detected in the brain, likely in telecephalon (Te) (A and B). At $3 \mathrm{dpf}$ larvae $(\mathrm{C}$ and $\mathrm{D})$ and $6 \mathrm{dpf}(\mathrm{E}$ and $\mathrm{F})$ the EGFP-positive neurons were more located in the ventral diencephalon (white lines) of the posterior tuberculum. Fluorescent images of adult zebra fish showing in vivo EGFP expression in the diencephalon $(\mathrm{G})$. No green fluorescent signal was noticed in wild-type zebra fish $(\mathbf{H})$. The following abbreviations are used: Olfactory bulb $(\mathrm{Ob})$, ventricular diencephalon (vDc), Pretectum (Pr), Caudal Hypothalamus (Hc), Telencephalon neurons (Te), Posterior tubercular neurons (Pt). Scale bar $=300 \mu \mathrm{m}(\mathrm{A}-\mathrm{F})$ and $3 \mathrm{~mm}(\mathrm{G}-\mathrm{H})$. (A, C and $\mathrm{E}$ - dorsal views and $\mathrm{B}, \mathrm{D}, \mathrm{F}-$ lateral views).

\section{Co-localization of TH in EGFP-positive neurons in posterior tuberculum}

In further assessments, we performed whole-mount in situ hybridization (WISH) assay using the th 1 gene probe in both $3 \mathrm{dpf}$ wild-type and transgenic larvae in order to evaluate whether the construct incorporation and GFP expression had a negative effect on the number of $\mathrm{TH}+$ neurons. Both wild-type (Figure 3A) and transgenic larvae (Figure 3B) were collected for in situ hybridization to compare the number of $\mathrm{TH}+$ cells. Results showed no significant difference in the number of $\mathrm{TH}+$ diencephalic cells between both groups $(p=0.777)$. Consistent with the distribution of th, EGFP expression in the brains of $3 \mathrm{dpf}$ larvae was mainly detected in vDC (Figure 3C). Based on these findings, we performed double-label immunohistochemistry using antibodies for TH and GFP to further determine the overlap of Tg (-2.5th:EGFP) with native TH expression on larvae and adult brains. Tyrosine Hydroxylase immunoreactive neurons (TH-ir) in the brain of transgenic larvae (6dpf) were mainly detected in the vDC (Figure 4B) and along in this region, cells were also EGFP-positive (Figure 4C). We observed that the double-positive cells were evenly distributed into clusters 2,3 , and $4 / 5$ and sparsely distributed into the clusters 1 and 6 (Figure 4D). A schematic diagram showing the position of these clusters within or in the vicinity of the PT is also included in Figure 4.

Whole-mount in situ hybridization showing $\mathrm{TH}$ expression at 3 days post-fertilization (dpf) wild-type larvae (A) and $\mathrm{Tg}(-$ 2.5th:EGFP) larvae (B). EGFP expression in 3 dpf larvae (wholemount immunohistochemistry) by confocal microscopy (C). Scale bar $=30 \mu \mathrm{m}$.

Schematic diagram showing the position of dopaminergic cells in clusters within or in the vicinity of the posterior tuberculum (PT) (A). Confocal microscopic longitudinal section for single THimmunoreactive (red) label (B) and for single EGFP-immunoreactive (green) label (C). Fluorescent double labeling for TH and EGFP (D). The DA neurons in yellow are found in clusters 1, 2, 3, 4/5 and 6 in the ventral diencephalon (vDC) (D). The use of GFP antibody in wildtype zebra fish, or GFP antibody without primary TH resulted in a lack of staining (data not shown). Scale bar $=30 \mu \mathrm{m}$. Images obtained from larvae at 6 days post-fertilization (dpf).

\section{6-OHDA neurotoxicity on Tg(-2.5th:EGFP) zebrafish}

Next, our transgenic line was used as an in vivo model to investigate the effects of 6-OHDA on neuronal survival and on the larvae and adult fish locomotor activity. Firstly, we examined the effects of treatment with $500 \mu \mathrm{M}$ of 6-OHDA on swimming activity of 6dpf larvae (Figure 5A). We observed marked alterations in the swimming activity of the treated larvae after treatment, i.e., the total distance moved significantly decreased approximately 2-fold compared with the untreated larvae $(n=8-17, p<0.001)$ (Figure $5 A)$. We also observed OHDA-induced alterations in the density of EGFP-positive neurons in larvae at $6 \mathrm{dpf}$. A reduction of $60 \%$ in green fluorescence was detected when compared with controls $(n=17, \mathrm{p}<0.05)$ (Figures 5B-5D).

Changes in the swimming activity of $6 \mathrm{dpf}$ larvae after treatment with 6-OHDA (A). The mean total distance travelled under a dark stimulus was examined by a video tracking system in untreated larvae (white bar) and in the treated counterparts (grey bar). Percentage of total EGFP intensity in the ventral diencephalon (vDC) of untreated and treated larvae at 6 days post-fertilization (dpf) (B). The bars represent mean \pm s.e.m. $\left({ }^{*} \mathrm{P}<0.05, * * \mathrm{P}<0.01\right)$. Fluorescent double labeling for TH (red) and GFP (green) in the vDC of untreated (control) (C) and treated (OHDA) larvae (D) assessed in confocal microscopic longitudinal sections.

The neurotoxin 6-OHDA also induced alterations in the locomotors activity in adult fish. Time-dependent changes in the locomotors activity of treated adult fish were detected throughout the 
experiment as shown in Figure 6A. At day 1, the individual activity of zebrafish, measured as tail-flips per minute, significantly decreased by $30 \%(48.4 \pm 10.6, n=18, p<0.05)$ after fish received the first dose of 6-OHDA. At day 3, lethargy was attenuated to values closed to the controls $(67.8 \pm 11.4, \mathrm{n}=10, \mathrm{p}>0.05)$ but this behavior was again intensified (44.6 $6 \pm 9.8, \mathrm{n}=8, \mathrm{p}<0.01)$ at day 6,3 days after fish received the second re-injection of the drug. At day 9 , the motor activity of treated fish returned to values observed in the controls. The activity of control fish was maintained constant throughout the experiment $(68.0 \pm 2.8, \mathrm{n}=49)$ with the exception at day 9 , in which the levels dropped to values of $55.8 \pm 9.9(\mathrm{n}=6, \mathrm{p}<0.05)$.

Alterations in the locomotors activity of adult zebra fish after 6-OHDA treatment are pictured in (A). Grey bars represent timedependent changes in treated fish after administration of $33 \mathrm{mg} / \mathrm{kg}$ 6-OHDA. The activity of zebra fish received only saline solution is represented in white bars. Fish activity was recorded using an electric biosensor. Bars show the mean with $95 \%$ confidence belts. Representative western blots showing the expression of Tyrosine hydroxylase (TH) (B) and Caspase-3 (C) in untreated (Control) and treated brains throughout experiment and the respective levels obtained by densitometry. Bars represent mean \pm s.e.m. Values are expressed as a ratio of the proteins to $\beta$-actin. Statistical differences between groups were checked by Student's t-test for independent means (* represent $\mathrm{P}<0.05$ ).

At the sub cellular level, native TH protein expression changed in a time- dependent manner (Figure 6B). Western blot analysis confirmed that in treated fish, the levels of TH protein significantly decreased by $38 \%(0.50 \pm 0.13$ arbitrary units, $n=4, p<0.05)$ compared with controls at day 1 , followed by an increase at days $6(0.71 \pm 0.1$ arbitrary units, $n=4)$ and $9(0.76 \pm 0.02$ arbitrary units, $n=4)$ compared with day 1 . However, the levels are maintained significantly lower than in controls $(0.87 \pm 0.03$ arbitrary units, $n=4, p<0.05)$ (Figure $6 \mathrm{~B})$. We further examined in the same blots the levels of caspase-3 (Figure 6C). Specifically, in treated brains, caspase-3 expression was strongly induced $(0.45 \pm 0.09, \mathrm{n}=4, \mathrm{p}<0.05)$ after the first i.m. injection of 6-OHDA at day 1, and a peak of protein expression could be demonstrated at day $6(0.62 \pm 0.1$ arbitrary units, $n=4 p<0.05), 3$ days after the second re-dosing. At day 9, the levels of caspase- 3 returned to values close to controls ( $0.35 \pm 0.06$ arbitrary units) (Figure 6C).

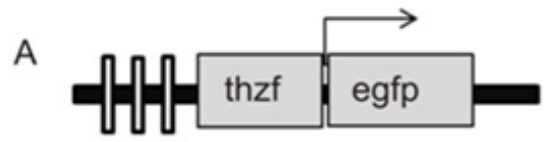

B
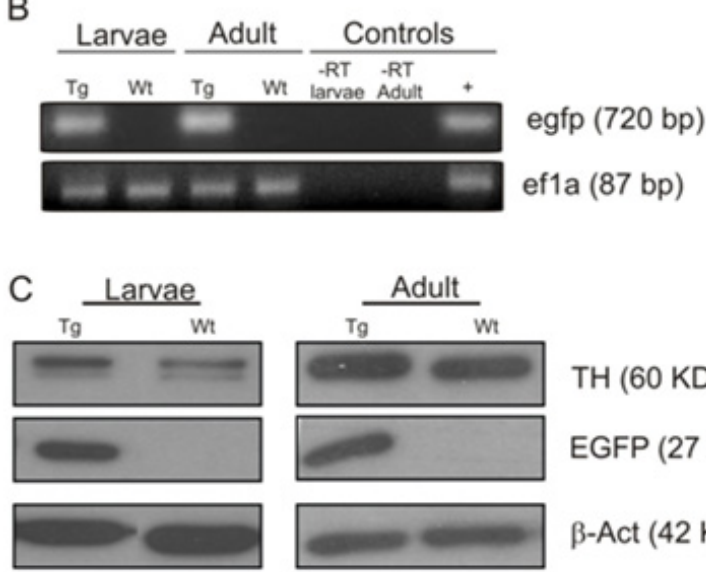

TH $(60 \mathrm{KDa})$

EGFP (27 KDa)

$\beta$-Act (42 KDa)

Figure I The generation of the $\operatorname{Tg}(-2.5$ th:EGFP) zebra fish line.
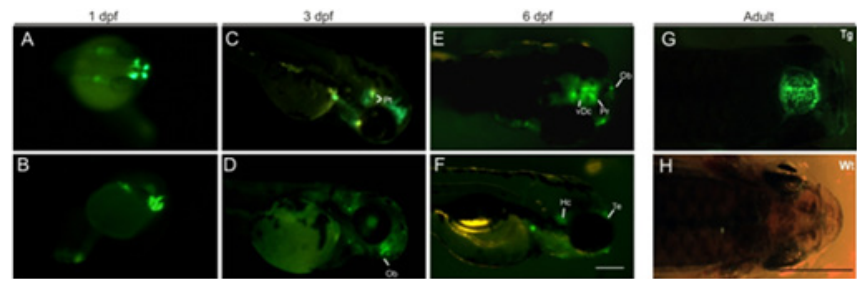

Figure 2 In vivo EGFP expression in Tg(-2.5th:EGFP) zebrafish.
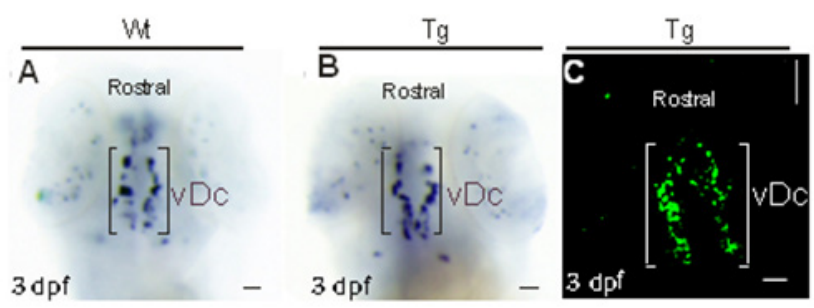

Figure 3 Comparison of tyrosine hydroxylase (TH) and EGFP expression in $\mathrm{Tg}(-2.5$ th:EGFP) larvae.
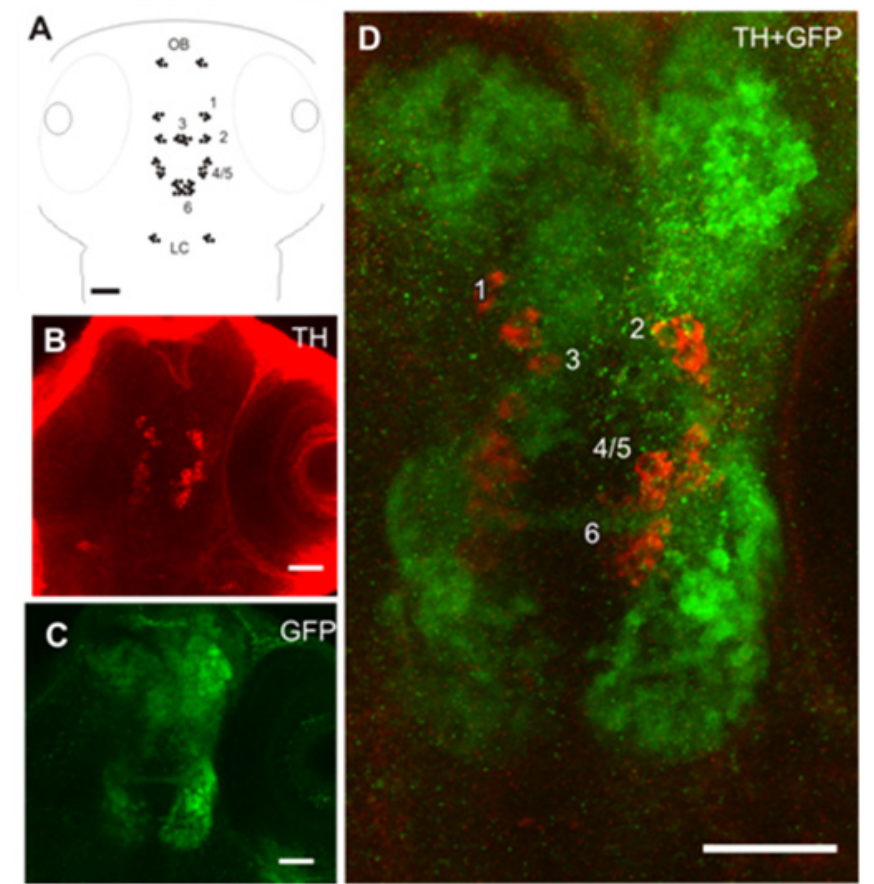

Figure 4 EGFP and tyrosine hydroxylase (TH) distribution in Tg(-2.5th:EGFP) larvae.

\section{Discussion}

The study of the molecular mechanisms associated with DAcell loss in models of PD has been complicated by the limitations of the existing animal models. Here, we report the development of a novel $\operatorname{Tg}(-2.5$ th:EGFP) zebrafish line in which the fluorescent reporter gene is driven by a $2.5 \mathrm{~kb}$ fragment of the zebrafish th 1 promoter. In zebrafish, the tyrosine hydroxylase promoter (from both rat and zebra fish) has been used to produce transgenic lines. However, these promoters have driven expression in retinal DA neurons. ${ }^{15,16} \mathrm{We}$ isolated an endogenous zebra fish th $l$ gene promoter to generate a transgenic zebra fish that expresses EGFP only in the brain. In our hands, this promoter drove gene expression in different clusters of neurons located into anterior and posterior groups along the PT of the ventral diencephalon. These cells project prominent descending axons through the diencephalon into the spinal cord, 
which is a pattern commonly seen in viable DA neurons of zebra fish brains. ${ }^{25}$ Our immunolabeling studies revealed that these neurons are $\mathrm{TH}$-ir cells by co-labeling with $\mathrm{TH}$ antibody. In previous studies, a larger 12-kb fragment of the same th1 gene promoter was used and demonstrated that it was specific for retinal dopaminergic cells in $\mathrm{Tg}(-$ 12th:MmGFP) zebra fish. ${ }^{16}$ In the line of these evidences, the EGFP expression in $\operatorname{Tg}(-2.5$ th:EGFP) is driven by the regulatory elements of the th1 gene that we suppose be expressed mainly in TH populations $2-4 / 5$ in the PT. These neuronal clusters in the zebra fish diencephalon are the equivalent of the mammalian substantia nigra, ${ }^{10}$ which may explain the severe loss of neurons in the vDC following 6-OHDA treatment.
A
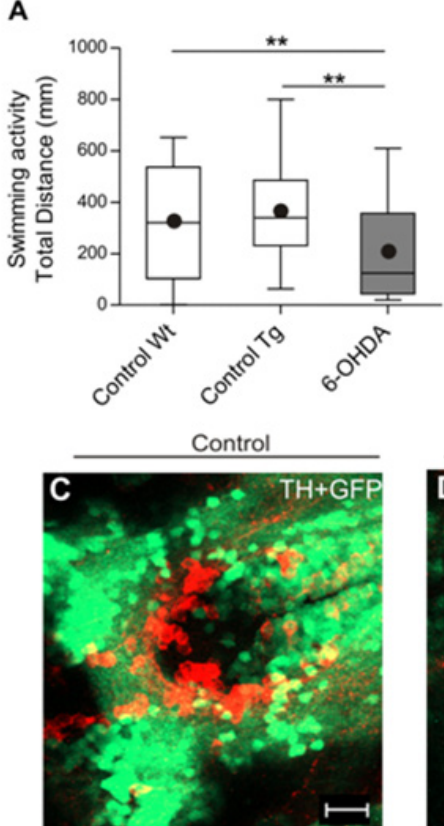

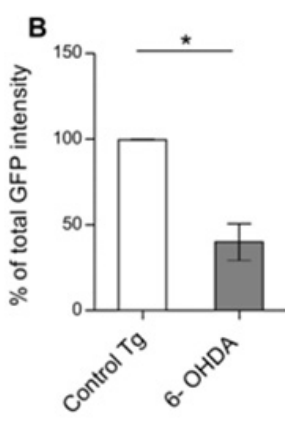

OHDA

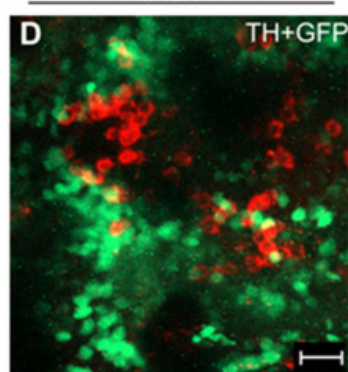

Figure 5 Effects of 6-OHDA (6-hydroxydopamine) in larvae of $\mathrm{Tg}(-2.5$ th: EGFP) zebrafish.

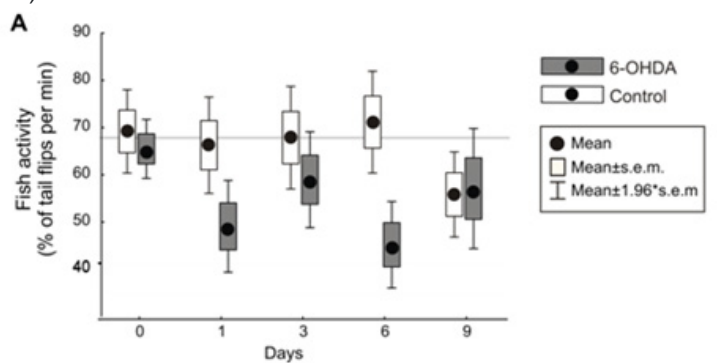

B

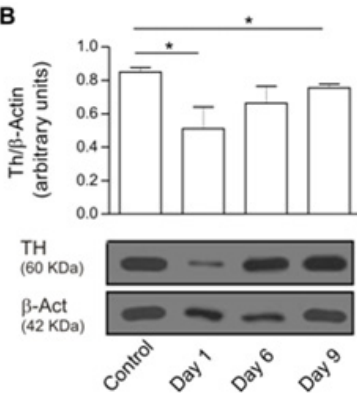

C

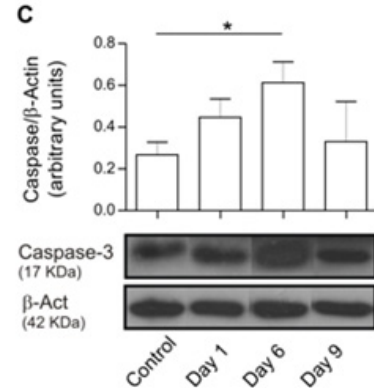

Figure 6 Effects of 6-OHDA (6-hydroxydopamine) in adult $\mathrm{Tg}(-2.5$ th:EGFP) zebrafish.

Although most neurons in the vDC expressed both EGFP and TH, we also detected cells that are EGFP-positive but TH-negative in the PT possibly due to the fact that these EGFP-positive neurons might be th2-positive neurons ${ }^{8,16}$ due to the genome duplication during teleost evolution. ${ }^{26}$ For this reason, the anti-TH antibody used in our study has a relatively lower affinity for the $\mathrm{TH} 2$ protein. In addition, we detected cells immunoreactive only for TH antibody mainly in adult brains, suggesting that our transgene is expressed, in other catecholaminergic cells not only in dopaminergic neurons. This fact is commonly seen in previous transgenic lines where the transgene is under regulation of regulatory elements from the tyrosine hydroxylase (th) gene. ${ }^{15,16}$

Since our promoter fragment induced gene expression in a neuronspecific dependent manner in our transgenic line, we further assessed the potential of this transgenic line for the study of neurotoxininduced DA cell loss as a model of PD. For this purpose, we used the dopaminergic neurotoxin, 6-OHDA. The effects of this drug, in contrast to MPTP, ${ }^{8,11}$ had not been studied in zebra fish expressing reporters in DA neurons. In our larvae, a concentration of $500 \mu \mathrm{M}$ of 6-OHDA induced loss of EGFP-positive neurons, and elicited marked deficits in the motor activity of fish. Similarly, the drug elicited similar effects on neuronal survival and on the swimming behaviors of adult fish. In particular, we detected a considerable decline in the expression of $\mathrm{TH}$ that was followed by increases in caspase- 3 expression in treated brains. We hypothesize that the lesions in putative DA neurons might interfere with the function of DA neurotransmission and consequently affect the motor behavior in zebra fish. However, this phenomenon seems to be reversible due to the tendency of DA neurons to recover by the end of the experiment. The tendency for the generality of catecholaminergic neurons to recover after treatment with 6-OHDA was previously reported in wild-type zebra fish and in other teleosts. ${ }^{12,27}$ The parkinsonian symptoms commonly seen in fish when they are treated with this neurotoxin ${ }^{12,28,29}$ can be explained by the high permeability of the blood brain barrier which is not often seen in mammalian models. ${ }^{30,31}$ However, the mechanism and targets of 6-OHDA action are relatively well known in vertebrates, i.e., this neurotoxin directly enters the neuronal processes via the catecholaminergic transporters due to its structural similarity with DA. Upon entry into the neurons, this neurotoxin can cause alteration in the cellular metabolism by interfering in the generation of reactive oxygen species and lead to cell death via apoptosis (reviewed in. ${ }^{30}$ ) Taken together, our data suggests the possibility of a similar mode of action in zebra fish DA neurons. In conclusion, our transgenic fish will be useful as a new model for the study of the life cycle of DA neurons and for high-throughput screening of molecules of interest for PD.

\section{Acknowledgements}

We thank to Lara Carvalho, Fish Facility Manager at Institute of Molecular Medicine (IMM, Portugal), for maintaining the zebra fish, António Temudo and Ana Nascimento from IMM for the assistance with the bioimaging analyses, Martin Scholze from Brunel University, U.K., for the assistance in the statistical data behavioral analyses and Ana Clara Cristovão from Burnett School of Biomedical Sciences, UCF, Florida, USA for the scientific discussion of the results regarding 6-OHDA experiments.

\section{Conflicts of interest}

None.

\section{Funding}

None.

\section{References}

1. Kruger R, Kuhn W, Muller T, et al. Ala30Pro mutation in the gene encoding alpha-synuclein in Parkinson's disease. Nat Genet. 1998;18(2):106-108. 
2. Zarranz JJ, Alegre J, Gomez Esteban JC, et al. The new mutation, E46K, of alpha-synuclein causes Parkinson and Lewy body dementia. Ann Neurol. 2004;55(2):164-173.

3. Yamamoto K, Ruuskanen JO, Wullimann MF, et al. Differential expression of dopaminergic cell markers in the adult zebrafish forebrain. J Comp Neurol. 2011;519(3):576-598.

4. Abeliovich A, Flint Beal M. Parkinsonism genes: culprits and clues. $J$ Neurochem. 2006;99(4):1062-1072.

5. Dehay B, Bezard E. New animal models of Parkinson's disease. Mov Disord. 2011;26(7):1198-1205.

6. Chesselet MF, Richter F. Modelling of Parkinson's disease in mice. Lancet Neurol. 2011;10(12):1108-1118.

7. Bandmann O, Burton EA. Genetic zebrafish models of neurodegenerative diseases. Neurobiol Dis. 2010;40(1):58-65

8. Xi Y, Yu M, Godoy R, et al. Transgenic zebrafish expressing green fluorescent protein in dopaminergic neurons of the ventral diencephalon. Dev Dyn.2011;240(11):2539-2547.

9. Flinn L, Mortiboys H, Volkmann K, et al. Complex I deficiency and dopaminergic neuronal cell loss in parkin-deficient zebrafish (Danio rerio). Brain. 2009;132(pt6):1613-1623.

10. Rink E, Wullimann MF. The teleostean (zebrafish) dopaminergic system ascending to the subpallium (striatum) is located in the basal diencephalon (posterior tuberculum). Brain Res. 2001;889(1-2):316-330.

11. Wen $\mathrm{L}$, Wei W, Gu W, et al. Visualization of monoaminergic neurons and neurotoxicity of MPTP in live transgenic zebrafish. Dev Biol. 2008;314(1):84-92.

12. Anichtchik OV, Kaslin J, Peitsaro N, et al. Neurochemical and behavioural changes in zebrafish Danio rerio after systemic administration of 6-hydroxydopamine and 1-methyl-4-phenyl-1,2,3,6-tetrahydropyridine. J Neurochem. 2004;88(2):443-453.

13. Lam CS, Korzh V, Strahle U. Zebrafish embryos are susceptible to the dopaminergic neurotoxin MPTP. Eur J Neurosci. 2005;21(6):1758-1762.

14. Sheng D, Qu D, Kwok KH, et al. Deletion of the WD40 domain of LRRK2 in Zebrafish causes Parkinsonism-like loss of neurons and locomotive defect. PLoS Genet. 2010;6:e1000914.

15. Gao Y, Li P, Li L. Transgenic zebrafish that express tyrosine hydroxylase promoter in inner retinal cells. Dev Dyn. 2005;233():921-929.

16. Meng S, Ryu S, Zhao B, et al. Targeting retinal dopaminergic neurons in tyrosine hydroxylase-driven green fluorescent protein transgenic zebrafish. Mol Vis. 2008;14:2475-2483.
17. Fujimoto E, Stevenson TJ, Chien CB, et al. Identification of a dopaminergic enhancer indicates complexity in vertebrate dopamine neuron phenotype specification. Dev Biol. 2011;352(2):393-404.

18. Westerfield M. The zebrafish book. Guide for the laboratory use of zebrafish (Danio rerio). (5th edn), University of Oregon Press, Eugene, USA. 1995. p.13848.

19. Kimmel CB, Ballard WW, Kimmel SR, et al. Stages of embryonic development of the zebrafish. Dev Dyn. 1995;203(3):253-310.

20. Kwan KM, Fujimoto E, Grabher C, et al. The Tol2kit: a multisite gateway-based construction kit for Tol2 transposon transgenesis constructs. Dev Dyn. 2007;236(11):3088-3099.

21. Bai Q, Mullett SJ, Garver JA, et al. Zebrafish DJ-1 is evolutionarily conserved and expressed in dopaminergic neurons. Brain Res. 2006;1113(1):33-44.

22. Thisse C, Thisse B. High-resolution in situ hybridization to wholemount zebrafish embryos. Nat Protoc. 2008;3(1):59-69.

23. Correia A, Cunha S, Scholze M, et al. A novel behavioral fish model of nociception for testing analgesics. Pharmaceuticals. 2011;4(4):665-680.

24. Cunha SR, Goncalves R, Silva SR, et al. An automated marine biomonitoring system for assessing water quality in real-time. Ecotoxicology. 2008;17(6):558-564.

25. Sallinen V, Torkko V, Sundvik M, Reenila I, Khrustalyov D, et al. (2009) MPTP and MPP+ target specific aminergic cell populations in larval zebrafish. J Neurochem 108(3): 719-731.

26. Candy J, Collet C. Two tyrosine hydroxylase genes in teleosts. Biochim Biophys Acta. 2005; 1727(1):35-44.

27. Contestabile A, Friz T, Caravaggio MV. Disappearance and recovery of catecholamine innervation in brain regions of adult goldfish following 6-hydroxydopamine treatment. Neurosci Lett. 1979;14(2-3):333-337.

28. Zhang ZJ, Cheang LC, Wang MW, et al. Ethanolic extract of fructus Alpinia oxyphylla protects against 6-hydroxydopamine-induced damage of PC12 cells in vitro and dopaminergic neurons in zebrafish. Cell Mol Neurobiol. 2012;32(1):27-40.

29. Wang M, Zhang Z, Cheang LC, et al. Eriocaulon buergerianum extract protects $\mathrm{PC} 12$ cells and neurons in zebrafish against 6-hydroxydopamineinduced damage. Chin Med. 2011;6:16.

30. Schober A. Classic toxin-induced animal models of Parkinson's disease: 6-OHDA and MPTP. Cell Tissue Res. 2004;318(1):215-224.

31. Deumens R, Blokland A, Prickaerts J. Modeling Parkinson's disease in rats: an evaluation of 6-OHDA lesions of the nigrostriatal pathway. Exp Neurol.2002;175(2):303-317. 